\title{
Magnetic noise in MgO-based magnetic tunnel junction rings
}

\author{
J. F. Feng, ${ }^{1, a)}$ Z. Diao, ${ }^{1}$ Gen Feng, ${ }^{1}$ E. R. Nowak, ${ }^{2}$ and J. M. D. Coey ${ }^{1}$ \\ ${ }^{1}$ CRANN and School of Physics, Trinity College, Dublin 2, Ireland \\ ${ }^{2}$ Department of Physics and Astronomy, University of Delaware, Newark, Delaware 19716, USA
}

(Received 6 December 2009; accepted 31 December 2009; published online 3 February 2010)

\begin{abstract}
Magnetization switching is investigated in ring-shaped $\mathrm{MgO}$-based magnetic tunnel junctions with $168 \%$ tunneling magnetoresistance. Besides the forward and reverse onion states, two vortex states and several metastable states are observed for the ferromagnetic free layer. Electrical noise is used to characterize the low frequency magnetization dynamics; a stationary $1 / f$ noise spectrum is observed within each magnetic state but they are separated by noise peaks which show a $1 / f^{2}$ spectrum that is associated with slow random telegraph fluctuations. In the $1 / f$ region, the normalized magnetic noise parameter, $\alpha_{\text {mag, }}$ is shown to be consistent with the fluctuation-dissipation theorem. (C) 2010 American Institute of Physics. [doi:10.1063/1.3295707]
\end{abstract}

Over the last decade, magnetic tunnel junctions (MTJs) have attracted much attention due to their potential application in spin electronic devices such as magnetoresistive random access memory (MRAM), magnetic field sensors, and logic devices. ${ }^{1,2}$ Junctions with bcc Fe-based electrodes and a (001)-MgO barrier have been reported to exhibit high tunneling magnetoresistance (TMR) approaching the theoretical maximum. ${ }^{3,4}$ TMR is usually defined as $\left(R_{\mathrm{AP}}-R_{\mathrm{P}}\right) / R_{\mathrm{P}}$, where $R_{\mathrm{P}}$ and $R_{\mathrm{AP}}$ are the resistances of a MTJ when the magnetizations of the two ferromagnetic (FM) electrodes are parallel and antiparallel, respectively.

For MTJs with conventional shapes, two stable magnetic configurations with well-defined switching between them can be easily achieved, corresponding to the parallel and antiparallel states of the MTJ as supposed in the definition. However, another stable magnetic state, namely the vortex state, may form in microstructured magnetic rings $^{5-8}$ and discs, ${ }^{9}$ in order to minimize the magnetostatic energy of the system. Ring-shaped magnets with larger diameters and ring width exhibit more complex metastable states. ${ }^{6,7}$ The ring geometry offers some advantages in MRAM applications due to its stability on reducing the memory element size ${ }^{10}$ However, most of the previous studies on magnetic rings were focused on a single magnetic layer ${ }^{5-8}$ or GMR spin-valves. ${ }^{9}$ There are few reports on MTJ rings, ${ }^{11,12}$ and none on MgO-based MTJ rings with high TMR.

In this letter, we investigate magnetization switching and low frequency noise in micron-scale $\mathrm{MgO}$-based MTJ rings with high TMR. During the magnetization switching of the free $\mathrm{CoFeB}$ layer, the magnetic state switches from the forward onion state, via a vortex state, to the reverse onion state, with other metastable states in between. We have observed stationary low-frequency noise with a $1 / f$ spectrum in all these states. The $1 / f$ noise is attributed to thermally activated magnetization fluctuations of the FM layer, which can be understood in terms of the fluctuation-dissipation theorem. ${ }^{13}$

Typical MgO-based MTJ rings consist of Ta 5/Ru 20/Ta $5 / \mathrm{Ni}_{81} \mathrm{Fe}_{19} 5 / \mathrm{Ir}_{22} \mathrm{Mn}_{78} 10 / \mathrm{Co}_{90} \mathrm{Fe}_{10}$ 2/Ru $0.85 / \mathrm{Co}_{40} \mathrm{Fe}_{40} \mathrm{~B}_{20}$ (CoFeB) 3/MgO 1.5/CoFeB 5/Ta 5/Ru 10 (from the bottom electrode to the top, with thickness given in nanometers).

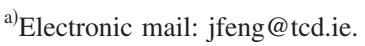

Further details on the growth process can be found in Ref. 14. The MTJ stack was patterned into circular rings with outer diameter of $2.6 \mu \mathrm{m}$, inner diameter of $1.0 \mu \mathrm{m}$, and width of $800 \mathrm{~nm}$ by electron beam lithography. A scanning electron microscope image of a ring is shown in the inset of Fig. 1. High vacuum annealing of the patterned junctions in the temperature range of $200<T_{\mathrm{a}}<400{ }^{\circ} \mathrm{C}$ was carried out in an applied magnetic field of $800 \mathrm{mT}$ for one hour. All magnetotransport measurements were made by a four-probe method. Electrical noise was measured at room temperature and under constant current bias using a commercial ac coupled low noise voltage amplifier (Stanford Research Systems SR552). The signal was further amplified; antialiasing filtered, and digitized using a 16-bit National Instruments DAQ card.

Due to the thin $\mathrm{MgO}$ barrier, resistance-area (RA) products of around $100 \Omega \mu \mathrm{m}^{2}$ are obtained for these MTJ rings (Fig. 1). Despite the low RA, a room temperature TMR value as high as $168 \%$ was achieved at $T_{\mathrm{a}}=350{ }^{\circ} \mathrm{C}$, which reflects the high quality of the $\mathrm{MgO}$ barrier. Figure 2(a) shows the $R-H$ loop near zero magnetic field for the MTJ ring annealed at $T_{\mathrm{a}}=300{ }^{\circ} \mathrm{C}$ and measured at a bias current $I=120 \mu \mathrm{A}$. The bottom $\mathrm{Co}_{90} \mathrm{Fe}_{10}$ and $\mathrm{CoFeB}$ layers are coupled across the Ru layer and pinned by the IrMn layer [Fig. 3(a)]. When scanning the magnetic field from positive to negative, the free $\mathrm{CoFeB}$ layer changes its magnetic state with field as shown in Figs. 3(b)-3(f). For all of the MTJ rings measured,

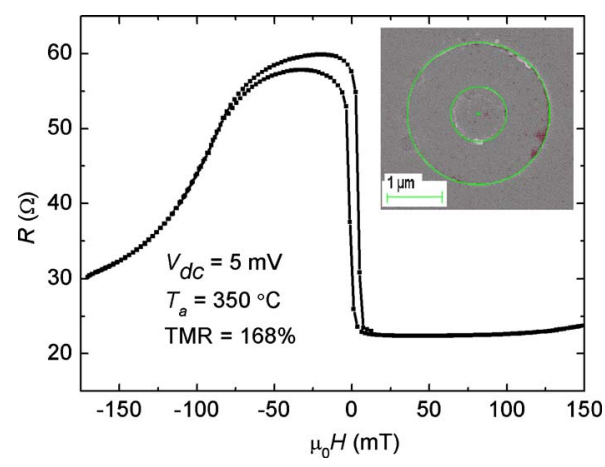

FIG. 1. (Color online) The resistance vs magnetic field loop for an MTJ ring with exchange bias. Inset is a scanning electron microscope image of the ring. 

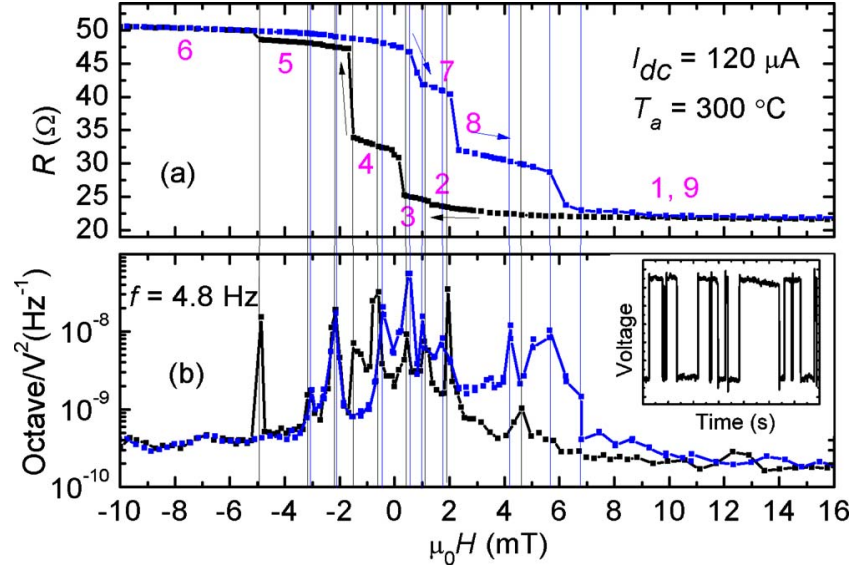

FIG. 2. (Color online) The magnetic field dependence of the MTJ ring resistance (a) and the normalized octave noise at $4.8 \mathrm{~Hz}$ (b). The lines show the noise peaks, and the numbers refer to the configurations in Figs. 3 and 4. The inset shows a time sequence of RTN at a noise peak.

dominant two-step behavior is evident, and a vortex state (clockwise or anticlockwise) and the intermediate state can be inferred from the resistance. Besides, four other metastable states are observed for this particular MTJ ring. The existence of the metastable states depends on the detailed geometrical parameters (outer diameter, ring width, and regularity) ${ }^{7}$ and the edge roughness of the microfabricated structure. Pinning sites, created during lift-off, may influence the magnetization process, altering the stability of the metastable states.

We further characterize the MTJ ring using the low frequency resistance noise spectrum as shown in Fig. 2(b) ${ }^{16-19}$ The noise is integrated across an octave centered at $4.8 \mathrm{~Hz}$. Two types of noise peaks occur during the magnetization switching. The first type occurs between two clearly defined magnetic states, e.g., between field position nos. 5 and 6 shown in Fig. 2(a). Another type of noise peak, often observed within a single magnetic state, e.g., around field position no. 4 , may reflect the occurrence of fractional vortices and composite domain walls, ${ }^{6,20}$ related to the microscopic domain rearrangement as shown in Figs. 3(c) and 3(e). At these peaks, there is stationary random telegraph noise [see the inset of Fig. 2(b)], with a $1 / f^{2}$ spectrum above a certain corner frequency - a noise spectrum with a Lorentzian-type character, it is due to thermally driven metastable reconfigurations in the domain structure of the free $\mathrm{CoFeB}$ layer. ${ }^{19}$ This explanation differs from that of Guo et al. ${ }^{18}$ who associated the $1 / f^{2}$ noise with magnetic after effect, which is a nonstationary process.

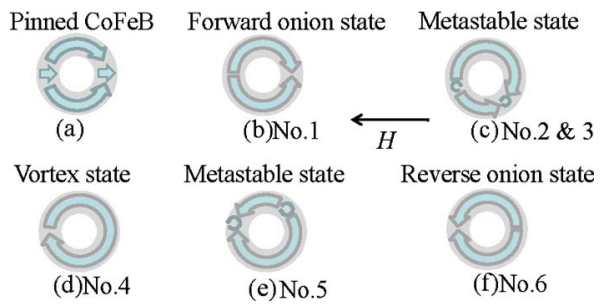

FIG. 3. (Color online) Schematic magnetization configurations for magnetic states of the pinned (a) and free [(b)-(f)] CoFeB layers in MTJ rings obtained as the field is scanned from positive to negative. The schematic is based on micromagnetic simulations using the oOMMF code (see Ref. 15).

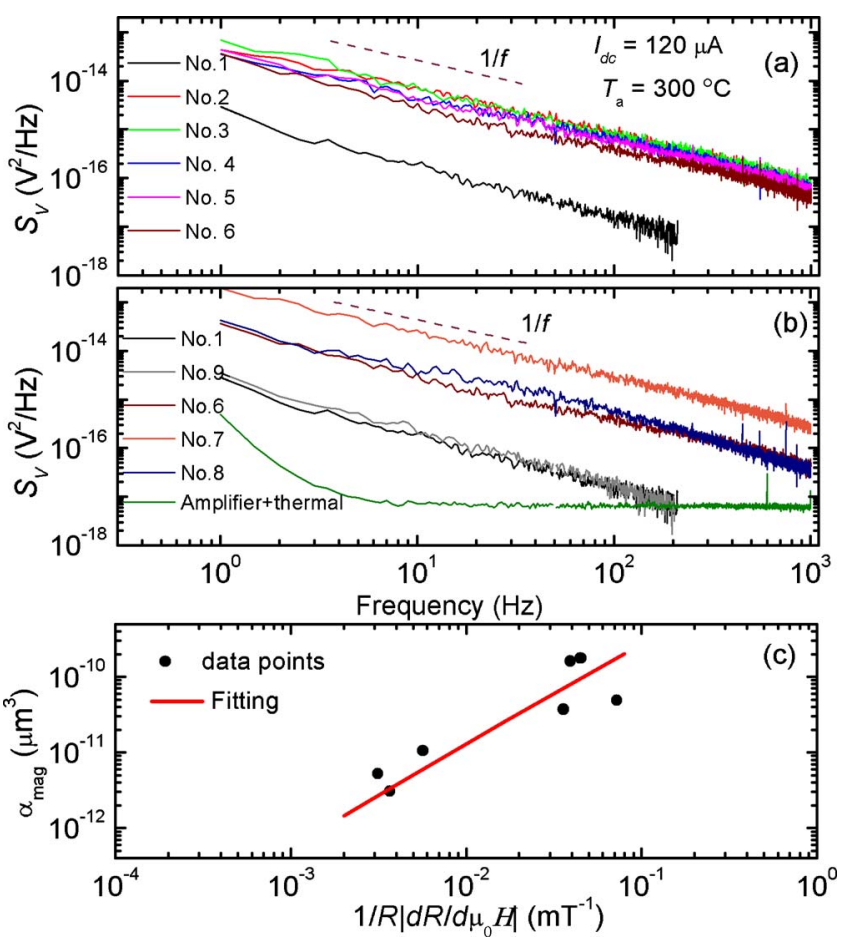

FIG. 4. (Color online) [(a) and (b)] show the power spectral density of the noise as a function of frequency at $I=120 \mu \mathrm{A}$, taken in different magnetic states marked in Fig. 2(a). (c) Shows the magnetic Hooge parameter $\alpha_{\text {mag }}$ plotted as a function of sensitivity; the line is a fit to the data.

Figure 4 plots the power spectral density of the MTJ ring in different, numbered magnetic states after thermal noise and amplifier noise have been subtracted. A $1 / f$ noise dependence at low frequencies is found when the free $\mathrm{CoFeB}$ layer is in any of its clearly defined magnetic states. The measurement is repeatable; as seen in the results at the same field position nos. 1 and 9. There is a higher noise level in the antiparallel state compared with the parallel state due to the resistance increase, besides the magnetic contribution of the fluctuating FM electrodes. Defining the normalized magnetic noise parameter $\alpha_{\mathrm{mag}}=S_{V}^{\prime} \Omega f / V^{2}$, where $\Omega$ is the free layer volume, $f$ the frequency, and $V$ the voltage applied to the MTJ ring, this parameter allows us to compare the noise level in different magnetic states. We plot $\alpha_{\text {mag }}$ against $\left|(1 / R) d R / d \mu_{0} H\right|$ in Fig. 4(c) for selected field positions on the $R-H$ curve. At high fields $(500 \mathrm{mT})$, only residual electrical noise is assumed to remain, which we subtracted from the measured power spectral density $S_{V}$ to obtain the magnetic contribution $S_{V}^{\prime}$. The $\alpha_{\mathrm{mag}}$ values in these MTJ rings are comparable to those reported by Ozbay et al. ${ }^{16}$ for $\mathrm{MgO}$ barrier MTJs with a conventional shape. $\alpha_{\text {mag }}$ in the forward and the reversed onion states are similar. A somewhat higher $\alpha_{\text {mag }}$ is obtained for the vortex and metastable states. A possible explanation could be nucleation of a vortex core in the ring. The vortex core moves perpendicular to the applied field during switching and is finally expelled from the ring. From this point of view, the higher $1 / f$ noise found around field position no. 7 shown in Fig. 2(a), near the magnetization switching between two magnetic states is due to incipient magnetic instability.

In thermal equilibrium, the fluctuation-dissipation theorem can be used to relate the magnetic resistance noise to the imaginary component of the ac susceptibility of the free 
layer $\chi_{R^{*}}^{\prime \prime}{ }^{13,21}$ Assuming the imaginary part is much smaller than the real part $\chi_{R}^{\prime}$, it can be shown that

$$
\alpha_{\mathrm{mag}}=\varepsilon(f, H) \frac{\mathrm{k}_{\mathrm{B}} T}{\pi \mu_{0} M_{s}} \frac{\Delta R}{R}\left(\frac{1}{R} \frac{d R}{d H}\right),
$$

where $\varepsilon(f, H)=\chi_{R}^{\prime \prime} / \chi_{R}^{\prime}$ is the ratio of the imaginary part of the ac susceptibility to the real part, $\mathrm{k}_{\mathrm{B}}$ is Boltzmann's constant, $T$ is the absolute temperature in Kelvin, $\mu_{0}$ is the permeability of free space $\left(4 \pi \times 10^{-7} \mathrm{~N} \mathrm{~A}^{-2}\right), M_{s}$ is the saturation magnetization of the FM layer, and $\Delta R$ is the total resistance change between parallel and antiparallel states. Equation (1) implies that there exists a relation between $\alpha_{\text {mag }}$ and $|(1 / R) d R / d H|$ which is linear if $\varepsilon$ is independent of field. This is indeed observed in our ring-shaped MTJs [linear fit in Fig. 4(c)]. The linear dependence has been previously reported in $\mathrm{AlO}_{\mathrm{x}}$-based MTJs, ${ }^{19,22,23}$ and in MgO-based MTJs with a TMR value of around $100 \%,{ }^{16}$ both patterned into a square shape. The calculated $\varepsilon(H)$ value for different magnetic states of the free $\mathrm{CoFeB}$ layer corresponds to a phase angle of $0.1^{\circ}-1^{\circ}$, which is similar to that reported by Ozbay et al. ${ }^{16}$ Our results on MTJ rings show that the fluctuationdissipation relation can be used to characterize the noise properties of MTJs with complicated domain structures.

In conclusion, micron-sized MTJ rings with a high TMR ratio $(\sim 170 \%)$ can be fabricated by electron beam lithography. Well defined onion and vortex states are observed, together with metastable states at intermediate magnetic fields. The low frequency magnetic resistance noise of $1 / f$ character arises from thermally activated magnetization fluctuations. Peaks where the noise is Lorentzian are related to slow stationary fluctuations. Our results suggest that the noise characteristics of $\mathrm{MgO}$-based $\mathrm{MTJ}$ rings will not preclude them from being used in multistate spin electronic devices, particularly if the formation of unstable domain structures can be avoided.

This work was supported by Science Foundation Ireland as part of the MANSE project, and the Ireland-China exchange agreement. Some support was also provided by En- terprise Ireland, as part of the "Spincurrents" FoNE network. E.R.N. also acknowledges partial support by DOE under Award No. DE-FG02-07ER46374.

${ }^{1}$ T. Miyazaki and N. Tezuka, J. Magn. Magn. Mater. 139, L231 (1995).

${ }^{2}$ J. S. Moodera, L. R. Kinder, T. M. Wong, and R. Meservey, Phys. Rev. Lett. 74, 3273 (1995).

${ }^{3}$ J. Mathon and A. Umersky, Phys. Rev. B 63, 220403 (2001).

${ }^{4}$ S. Ikeda, J. Hayakawa, Y. Ashizawa, Y. M. Lee, K. Miura, H. Hasegawa, M. Tsunoda, F. Matsukura, and H. Ohno, Appl. Phys. Lett. 93, 082508 (2008).

${ }^{5}$ J. Rothman, M. Kläui, L. Lopez-Diaz, C. A. F. Vaz, A. Bleloch, J. A. C. Bland, Z. Cui, and R. Speaks, Phys. Rev. Lett. 86, 1098 (2001).

${ }^{6}$ M. Steiner and J. Nitta, Appl. Phys. Lett. 84, 939 (2004).

${ }^{7}$ M. Kläui, C. A. F. Vaz, J. A. C. Bland, L. J. Heyderman, C. David, E. H. C. P. Sinnecker, and A. P. Guimarães, J. Appl. Phys. 95, 6639 (2004).

${ }^{8}$ J. Llandro, T. J. Hayward, D. Morecroft, J. A. C. Bland, F. J. Castaño, I. S. Coin, and C. A. Ross, Appl. Phys. Lett. 91, 203904 (2007)

${ }^{9}$ R. P. Cowburn, D. K. Koltsov, A. O. Adeyeye, M. E. Welland, and D. M. Tricker, Phys. Rev. Lett. 83, 1042 (1999).

${ }^{10}$ J.-G. Zhu, Y. F. Zheng, and G. A. Prinz, J. Appl. Phys. 87, 6668 (2000).

${ }^{11}$ C. C. Chen, C. Y. Kuo, Y. C. Chang, C. C. Chang, L. Horng, T. Wu, G. Chern, C. Y. Huang, M. Tsunoda, M. Takahashi, and J. C. Wu, J. Magn. Magn. Mater. 310, 1900 (2007).

${ }^{12}$ H. X. Wei, M. C. Hickey, G. I. R. Anderson, X. F. Han, and C. H. Marrows, Phys. Rev. B 77, 132401 (2008).

${ }^{13}$ H. T. Hardner, M. B. Weissman, M. B. Salamon, and S. S. P. Parkin, Phys. Rev. B 48, 16156 (1993).

${ }^{14}$ J. F. Feng, G. Feng, J. M. D. Coey, X. F. Han, and W. S. Zhan, Appl. Phys. Lett. 91, 102505 (2007).

${ }^{15}$ M. J. Donahue and D. G. Porter, OOMMF User's Guide, Version 1.2 Alpha 3 (National Institute of Standards and Technology, Gaithersburg, MD, 2002).

${ }^{16}$ A. Ozbay, A. Gokce, T. Flanagan, R. A. Stearrett, E. R. Nowak, and C. Nordman, Appl. Phys. Lett. 94, 202506 (2009).

${ }^{17}$ J. Scola, H. Polovy, C. Fermon, M. Pannetier-Lecoeur, G. Feng, K. Fahy, and J. M. D. Coey, Appl. Phys. Lett. 90, 252501 (2007).

${ }^{18}$ F. Guo, G. McKusky, and E. D. Dahlberg, Appl. Phys. Lett. 95, 062512 (2009).

${ }^{19}$ L. Jiang, E. R. Nowak, P. E. Scott, J. Johnson, J. M. Slaugher, J. J. Sun, and R. W. Dave, Phys. Rev. B 69, 054407 (2004).

${ }^{20}$ O. Tchernyshyov and G. W. Chern, Phys. Rev. Lett. 95, 197204 (2005).

${ }^{21}$ N. Smith, A. M. Zeltser, D. L. Yang, and P. V. Koeppe, IEEE Trans. Magn. 33, 3385 (1997).

${ }^{22}$ C. Ren, X. Y. Liu, B. D. Scharg, and G. Xiao, Phys. Rev. B 69, 104405 (2004)

${ }^{23}$ S. Ingvarsson, G. Xiao, S. S. P. Parkin, W. J. Gallagher, G. Grinstein, and R. H. Koch, Phys. Rev. Lett. 85, 3289 (2000). 